\title{
Tipo de parto e aleitamento precoce: impacto no estado nutricional da criança com 24 meses
}

\author{
Type of delivery and early breastfeeding: impact on the nutritional \\ status of 24-month-old child
}

\begin{abstract}
Rayane Quites Senra, ${ }^{1}$ Isabel Rey Madeira, ${ }^{1}$ Fabiana Migliaccio Mansur, ${ }^{1}$ Nádia Cristina Pinheiro Rodrigues, ${ }^{1}$ Antônia da Conceição Cylindro Machado, ${ }^{1}$ Celise Regina Alves da Motta Meneses, ${ }^{1}$ Vinicius Anciães Darriba, ${ }^{1}$ Ana Paula Córdova da $\operatorname{Costa}^{1}$ e Simone Augusta Ribas ${ }^{1}$
\end{abstract}

'Universidade do Estado do Rio de Janeiro

\section{Palavras-chave:}

Parto normal; Microbiota; Cesárea; Aleitamento materno; Estado nutricional; Sobrepeso.

\section{Resumo}

Introdução: os primeiros mil dias de vida compõem um período de janela de oportunidades no qual o corpo é modulado para responder a estímulos externos, podendo determinar agravos à saúde ainda na infância, como o desenvolvimento de excesso de peso. Objetivo: analisar o impacto do número de consultas pré-natais, o tipo de parto e o aleitamento precoce no estado nutricional antropométrico de crianças de 24 meses. Métodos: estudo descritivo, longitudinal e observacional das 58 crianças que permaneceram na coorte até os 2 anos. As crianças foram classificadas quanto ao estado nutricional e alocadas nos grupos com e sem excesso de peso. Foram analisadas, dentro dos grupos, variáveis categóricas que pudessem influenciar o estado nutricional. Foi aplicado modelo de regressão logística utilizando como variável dependente excesso de peso e como variáveis independentes número de consultas médicas, tipo de parto e aleitamento materno antes de 6 horas de vida. Resultados: das variáveis analisadas, apenas o tipo de parto cesárea e a ausência de aleitamento materno antes de 6 horas de vida se associaram positivamente com o excesso de peso aos 2 anos de idade (p-valor: 0,01 e 0,03 respectivamente). Conclusões: o trabalho indica que o tipo de parto e o aleitamento materno precoce interferem na saúde da criança. A importância destas variáveis não se restringe ao estado nutricional e poderá ser melhor explicada por estudos futuros.

\section{Keywords:}

Natural childbirth; Microbiota; Cesarean section; Breast feeding; Nutritional status; Overweight.

\footnotetext{
Abstract

Introduction: the first thousand days of life make up a window of opportunities in which the body is modulated to respond to external stimuli, and can determine health problems in childhood, such as the development of excess weight. Objective: to analyze the impact of the number of prenatal consultations, the type of delivery and early breastfeeding in the anthropometric nutritional status of 24-month-old children. Methods: descriptive, longitudinal and observational study of 58 children who remained in the cohort up to two years. The children were classified by nutritional status and allocated in the groups with and without excess weight. Within the groups, categorical variables that could influence nutritional status were analyzed. A logistic regression model was applied, using overweight as the dependent variable, and, as independent variables, the number of medical visits, type of delivery, and breastfeeding before six hours of life. Results: from the analyzed variables, only the type of cesarean delivery and the absence of breastfeeding before six hours of life were positively associated with overweight at two years of age (p: 0.01 and 0.03 , respectively). Conclusions: the work indicates that the type of childbirth and early breastfeeding interfere with the health of the child. The importance of these variables is not restricted to nutritional status and may be better explained by future studies.
} 


\section{INTRODUÇÃO}

O excesso de peso é um problema de saúde pública prevalente não só na fase adulta como também na infância. No Brasil, em 2017, foi observado pelo Sistema de Vigilância Alimentar e Nutricional (Sisvan) do Ministério da Saúde que $40,31 \%$ das crianças entre 0 e 2 anos de idade encontraram-se com classificação excesso de peso, sendo a prevalência do risco de sobrepeso de $21,72 \%$, sobrepeso de $10,57 \%$ e obesidade $8,02 \%$. ${ }^{1}$

Levando em conta a alta prevalência de crianças de 0 a 2 anos com excesso de peso, o presente estudo buscou analisar variáveis referentes ao período pré-natal, ao nascimento e ao aleitamento materno para estudar a influência delas sobre o excesso de peso.

Foi realizado um estudo prospectivo de coorte. As crianças foram captadas e seguidas até 2 anos de idade. O corte de 2 anos de idade levou em conta a janela de oportunidade de influência na saúde descrita na literatura como o conceito dos primeiros mil dias de vida, que se estende da concepção até os 24 meses. ${ }^{2}$

Os primeiros mil dias de vida são muito importantes, pois é nesse período que os indivíduos são expostos a estímulos externos pela primeira vez após o nascimento e o corpo é modulado para responder a esses estímulos, podendo determinar agravos à saúde ainda na infância e predisposição para doenças crônicas na fase adulta. ${ }^{3}$

Ao longo da vida intrauterina e dos primeiros mil dias de vida, existem fatores que funcionam como influenciadores de um adequado desenvolvimento do feto e da criança. Alguns desses fatores são: alimentação materna, tipo de parto, alimentação infantil, incluindo o aleitamento materno, e fatores genéticos, sendo este último um fator fixo que pode, no entanto, ser moldado pelo meio ambiente, como tem sido estudado. ${ }^{3}$

A nutrição tem dupla carga no desenvolvimento e crescimento infantil, sendo a desnutrição crônica e deficiência de nutrientes essenciais tão perigosos quanto o problema crescente da exposição infantil aos alimentos não adequados desde a primeira infância, como as fórmulas lácteas com elevado teor de carboidratos, gorduras e proteínas, baixo teor de oligossacarídeos e alimentos industrializados. ${ }^{4,5}$

O leite materno é considerado o melhor alimento para nutrição infantil, pois contém a quantidade de nutrientes essenciais, sofrendo mudanças na composição condizentes à necessidade em cada fase do desenvolvimento infantil. O colostro é a primeira composição do leite materno produzido e importante fonte de nutrientes que fornecem proteção contra infecções e doenças imunomediadas e estimula a maturação do sistema imune da mucosa intestinal. ${ }^{6}$ Sendo assim, o aleitamento materno iniciado o mais cedo possível fornece benefícios à saúde do lactente.

O leite materno é composto por elementos bioativos e com propriedades anti-inflamatórias, além de conter anticorpos que se mantêm ativos ao longo do trato gastrointestinal do recém-nascido, protegendo-o contra micro-organismos patogênicos que causam disbiose. O colostro e o leite materno são fontes contínuas de bactérias comensais, potencialmente probióticos para o intestino do lactente. ${ }^{7}$ A colonização da microbiota intestinal ocorre ao longo dos 2 primeiros anos de vida. Uma microbiota simbiótica e com interações microbianas é essencial para o desenvolvimento imunológico e metabólico do organismo, sendo a disbiose e a redução da diversidade microbiológica fatores de risco para doenças sistêmicas na infância, como a obesidade. O leite materno promove uma oferta contínua de bactérias simbióticas para o intestino do lactente e moléculas que agem como prebióticos, os oligossacarídeos. ${ }^{6}$

O processo de colonização da microbiota do neonato é favorecido pelo início precoce do aleitamento materno, ainda nas primeiras horas de vida, sendo esta uma das recomendações de boas práticas de amamentação da Organização Mundial da Saúde (OMS). ${ }^{8}$ Outro importante benefício do início da amamentação nas primeiras horas de vida são maiores taxas e duração do aleitamento materno ao longo dos primeiros meses de vida do lactente. Isso porque o contato precoce pele-pele entre mãe e neonato, propiciado pelo aleitamento materno, está relacionado com indução de comportamentos inatos do recém-nascido e da mãe que levam à amamentação bem-sucedida. Esse contato íntimo permite desenvolver um padrão de interação recíproca e sincronizada, sendo fatores hormonais e psicológicos da mãe essenciais nesse processo. Para as mães, este contato pele-pele nas primeiras horas de vida resulta na construção de vínculo e apego que proporciona mais domínio e autoconfiança para amamentar. Todos estes fatores são fundamentais para o sucesso do aleitamento ao longo dos primeiros meses de vida da criança. ${ }^{9}$

Além do início do aleitamento materno em momento oportuno, sua manutenção de forma exclusiva até os 6 meses de vida e de forma não exclusiva até os 24 meses também se mostram benéficos, sendo relacionados à redução na morbimortalidade por diarreias, infecções respiratórias, diminuição do risco de obesidade na infância e de diabetes, hipercolesterolemia e hipertensão arterial sistêmica na adultícia. ${ }^{10}$ Nesse sentido, ressalta-se a importância da orientação ao aleitamento materno 
durante o pré-natal e na maternidade, buscando informar e orientar as futuras mães quanto à importância dessa prática. Além disso, é essencial que a orientação e o apoio ao aleitamento materno continuem ao longo dos 2 primeiros anos de idade da criança.

O tipo de parto é descrito na literatura como um importante influenciador do desenvolvimento do indivíduo. A cesárea implica de modo negativo na saúde de lactentes e pode predispor problemas de saúde como asma, alergias, diabetes mellitus tipo 1, doença celíaca, sobrepeso e obesidade, que podem se manifestar da infância à idade adulta. ${ }^{11}$

Durante o parto vaginal, o neonato é exposto à microbiota materna vaginal e fecal, levando à colonização por bactérias benéficas ao trato gastrointestinal, que proporcionarão uma menor probabilidade de disbiose, o que possivelmente seria um fator protetor contra a obesidade. Crianças nascidas de parto tipo cesárea apresentam nas primeiras 24 horas de vida microbiota fecal com maior similaridade com a microbiota da pele materna e com a presença de mais bactérias patogênicas quando comparadas a crianças nascidas de parto vaginal. ${ }^{12}$

Além de influenciar a composição da microbiota intestinal, o tipo de parto também está relacionado a mudanças na composição do leite materno. O conteúdo do leite de mães de neonatos nascidos de parto vaginal e de cesárea não eletiva apresentam composição semelhante e equilibrada de agentes probióticos e prebióticos, ao contrário do encontrado no leite de mães submetidas a cesáreas eletivas, nas quais o microbioma do leite materno se encontra alterado. Essa diferença pode ser encontrada do colostro aos seis meses pós-natais, sendo o lactente, possivelmente, exposto a um maior fator de risco para obesidade. ${ }^{13}$

Neste contexto, partindo-se da hipótese de que o período dos mil dias é uma janela de oportunidade e modulação de fatores metabólicos e microbiológicos, o presente estudo teve como objetivo avaliar o grau de influência de variáveis relacionadas ao período pré-natal, ao parto e à alimentação infantil até os 2 anos de idade sobre o desenvolvimento de excesso de peso em crianças aos 2 anos de idade.

\section{MÉTODO}

Trata-se de um estudo longitudinal e observacional, de revisão de prontuário, das crianças da coorte do projeto Assistência Multidisciplinar em Pediatria (Amar), aos 2 anos de idade. O Amar é um projeto de extensão universitária do Departamento de Pediatria da Faculdade de Ciências Médicas da Universidade do
Estado do Rio de Janeiro (FCM-Uerj), de cunho multidisciplinar, cujo cenário é o Ambulatório de Pediatria do Hospital Universitário Pedro Ernesto da Uerj (HupeUerj). O Amar iniciou-se em 2007 com o objetivo de aprimorar assistência, ensino e pesquisa em atenção primária à criança.

O Hupe-Uerj tem o título de Hospital Amigo da Criança, conferido pela OMS a todos os que cumprem os passos por ela preconizados para incentivo ao aleitamento materno. ${ }^{14} \mathrm{O}$ projeto Amar focaliza a criança enquanto ser integral e integrado a partir de uma assistência na prevenção de agravos, promoção e educação em saúde, valorizando a multidisciplinaridade.

As crianças que participaram do presente estudo nasceram no Núcleo Perinatal do Hupe-Uerj a termo e não apresentaram risco perinatal. Após a alta do Núcleo Perinatal, essas crianças passaram a ser acompanhadas no Ambulatório de Pediatria Geral do Hupe-Uerj até os 5 anos de idade.

Do total de 350 crianças matriculadas no projeto de extensão Amar, 106 tinham dados completos. A totalidade de crianças teve Termo de Consentimento Livre e Esclarecido assinado pelo responsável legal, para participação no projeto de extensão. Essas crianças eram submetidas à avaliação clínica completa, segundo protocolo multidisciplinar de atendimento centrado na monitorização do crescimento e desenvolvimento, alimentação, prevenção e diagnóstico das principais doenças da infância. As crianças com três ou mais faltas consecutivas eram excluídas do estudo. O total de 81 e 58 crianças permaneceram no estudo até 1 e 2 anos, respectivamente.

As crianças que completaram 2 anos de idade no projeto foram classificadas, segundo a OMS, quanto ao estado nutricional. Para tal classificação, a OMS recomenda o referencial de índice de massa corporal (IMC) por idade e sexo e considera, para uma criança de 2 anos de idade:

a) magreza extrema - para escores-z de IMC menores que -3 desvios-padrão (DP);

b) magreza - para escores-z maiores ou iguais a -3 DP e menores que -2 DP;

C) eutrofia - para escores- $z$ maiores ou iguais a -2 DP e menores ou iguais a +1 DP;

d) risco para sobrepeso - para escores-z maiores que +1 DP e menores ou iguais a +2 DP;

e) sobrepeso - para escores-z maiores que $+2 \mathrm{DP}$ e menores ou iguais a +3 DP;

f) obesidade - para escores-z maiores que +3 DP.

O cálculo do escore-z do IMC foi feito a partir do software WHO Anthro (version 3.2.2, World Health Organization, Geneva, Switzerland), usando-se dados 
obtidos por meio das avaliações nutricionais antropométricas (peso e comprimento), da idade em meses e do sexo. ${ }^{15}$

As crianças foram pesadas sem sapatos e com roupas leves em balança da marca Filizola (São Paulo, SP, Brasil), com resolução do peso de 100 gramas, e suas estaturas foram medidas sem sapatos, em estadiômetro de parede do tipo Halpender-Holtain da marca Tonelli (Tonelli, Criciúma, SC, Brasil), com resolução de $1 \mathrm{~mm}$.

Os dados coletados foram arquivados em planilhas Excel versão 7 (MapInfo Corporation, Troy, NY, USA). As análises estatísticas foram feitas através do pacote estatístico R-Project versão 3.4.1 (The R Foundation for Statistical Computing, Vienna, Áustria).

Foram calculadas, para o grupo estudado, as frequências das seguintes variáveis categóricas: sexo, tipo de parto, idade gestacional de 40 semanas ou mais, aleitamento materno antes das 6 horas de vida, ${ }^{16}$ uso de fórmula láctea na maternidade, uso de fórmula inadequada no $1^{\circ}$ ano de vida, número de consultas pré-natais maior ou igual a seis, nutricionais maior ou igual a oito e médicas maior ou igual a dez,,$^{17,18}$ tempo de aleitamento materno exclusivo de quatro meses ou mais, tempo de aleitamento materno de 12 meses ou mais e tempo de aleitamento materno igual a 24 meses.

A amostra completa dos participantes foi subdividida em dois grupos - A e B. O grupo A foi constituído por crianças cujos estados nutricionais eram com risco de sobrepeso e sobrepeso. No grupo B, foram alocadas as crianças eutróficas e a criança com magreza, levando em conta que mesmo com seu z-IMC sendo -2,73, era uma criança saudável, não apresentando desnutrição ou outra doença. Foram comparadas frequências das variáveis categóricas estudadas, entre os grupos A e B, utilizando teste qui-quadrado e teste de Fisher. Calcularam-se também as razões de chances (OR) brutas para excesso de peso.

Utilizou-se regressão logística múltipla, tendo como variável dependente o grupo $\mathrm{A}$, referente às crianças com risco de sobrepeso e sobrepeso; e como variáveis independentes o tipo de parto e o aleitamento materno antes de 6 horas de vida. Para evitar colinearidade na especificação do modelo ajustado, avaliou-se previamente a associação entre aleitamento materno antes de 6 horas de vida e recebimento de fórmula láctea na maternidade. Foi encontrado p-valor igual a 0,008 no modelo logístico empregado para avaliar a associação entre essas variáveis.

Avaliou-se também a associação entre tipo de parto e aleitamento materno antes de 6 horas de vida, não sendo encontrada colinearidade entre essas variáveis.

A seleção das variáveis do modelo logístico de regressão múltipla se baseou na literatura científica e nos resultados da análise bivariada. Foram incluídas no modelo as variáveis com p-valor menor que 0,25 na análise bivariada, sendo excluídas as covariáveis fortemente correlacionadas. Considerando que as variáveis 1) aleitamento materno antes de 6 horas de vida e 2) receber fórmula na maternidade estavam fortemente correlacionadas, optou-se por incluir apenas a variável "aleitamento materno antes das 6 horas de vida" no modelo final.

O modelo final utilizou como variável dependente o grupo A e como variáveis independentes, número de consultas médicas ( $<10$, ou 10 ou mais consultas), tipo de parto e aleitamento materno antes de 6 horas de vida. Considerou-se significância estatística p-valor menor que 0,05 .

Este estudo foi aprovado pelo comitê de Ética e Pesquisa do Hupe-Uerj, onde está cadastrado sob o número: 2830/2010, CAAE n. 0277.0.228.000-10.

\section{RESULTADOS}

Foram estudadas as 58 crianças que completaram 2 anos na coorte, sendo a distribuição segundo seus estados nutricionais: 31 (53,5\%) eutróficos; um (1,7\%) com magreza; 18 (31\%) com risco de sobrepeso; e 8 (13,8\%) com sobrepeso. O grupo A foi composto por 26 crianças $(44,8 \%)$ e o grupo B por 32 crianças $(55,2 \%)$.

No grupo A, 13 (50\%) eram do sexo masculino e 13 $(50 \%)$ do sexo feminino. No grupo B, 17 (53,1\%) eram do sexo masculino e $15(46,9 \%)$ do sexo feminino. Os grupos não apresentaram diferenças estatisticamente significativas quanto às frequências de sexo $(p=0,81)$.

Ao se comparar as frequências das variáveis clínicas e metabólicas entre os grupos A e B, encontrou-se diferença estatisticamente significativa $(p<0,05)$ quanto ao tipo de parto (vaginal ou cesárea) e ter ou não recebido leite materno antes de 6 horas de vida.

Comparando-se as frequências das consultas entre os grupos com e sem excesso de peso, a frequência de seis ou mais consultas de pré-natal, quatro ou mais consultas de nutrição, dez ou mais consultas médicas foram $92,3 \%, 38,5 \%$ e $30,8 \%$, respectivamente, para grupo A e $96,6 \%, 50,0 \%$ e $46,9 \%$, respectivamente, para grupo B (Tabela 1).

Apenas o tipo de parto cesárea e a ausência de aleitamento materno antes de 6 horas de vida se associaram positivamente com o risco de sobrepeso e sobrepeso aos 2 anos de idade com significância estatística (p-valor: 
Tabela 1 - Comparação entre crianças de 2 anos com e sem excesso de peso em relação às frequências das variáveis categóricas estudadas, razões de chance e intervalos de confiança

\begin{tabular}{|c|c|c|c|c|c|c|}
\hline Variáveis categóricas & & Grupo A (n) & Grupo B (n) & OR bruto & IC $95 \%$ & p-valor \\
\hline \multirow[t]{2}{*}{ Sexo } & M & $50 \%(13)$ & $53,1 \%(17)$ & 0,9 & $0,3-2,5$ & 0,81 \\
\hline & $\mathrm{F}$ & $50 \%(13)$ & $46,9 \%(15)$ & & & \\
\hline \multirow[t]{2}{*}{ Fórmula na maternidade } & Sim & $36,4 \%(8)$ & $13,3 \%(4)$ & 3,7 & $1,0-14,5$ & 0,06 \\
\hline & Não & $63,6 \%(14)$ & $86,7 \%(26)$ & & & \\
\hline \multirow[t]{2}{*}{ Fórmula inadequada no $1^{\circ}$ ano } & Sim & $52 \%(13)$ & $41,9 \%(13)$ & 1,5 & $0,5-4,3$ & 0,45 \\
\hline & Não & $48 \%(12)$ & $58,1 \%(18)$ & & & \\
\hline \multirow[t]{2}{*}{ Tipo de parto } & C & $53,8 \%(14)$ & $21,9 \%(7)$ & 4,2 & $1,3-13,0$ & 0,01 \\
\hline & V & $46,2 \%(12)$ & $78,1 \%(25)$ & & & \\
\hline \multirow[t]{2}{*}{ N. de consultas no pré-natal } & $\geq 6$ & $92,3 \%(24)$ & $96,6 \%(28)$ & 0,4 & $0,1-5,0$ & 0,50 \\
\hline & $<6$ & $7,7 \%(2)$ & $3,4 \%(1)$ & & & \\
\hline \multirow[t]{2}{*}{ N. de consultas nutricionais } & $\geq 4$ & $38,5 \%(10)$ & $50 \%(16)$ & 0,6 & $0,2-1,8$ & 0,38 \\
\hline & $<4$ & $61,5 \%(16)$ & $50 \%(16)$ & & & \\
\hline \multirow[t]{2}{*}{ N. de consultas médicas } & $\geq 10$ & $30,8 \%(8)$ & $46,9 \%(15)$ & 0,5 & $0,2-1,5$ & 0,21 \\
\hline & $<10$ & $69,2 \%(18)$ & $53,1 \%(17)$ & & & \\
\hline \multirow[t]{2}{*}{ AM antes de $6 \mathrm{~h}$ de vida } & $\operatorname{Sim}$ & $69,2 \%(18)$ & $93,8 \%(30)$ & 0,2 & $0,0-0,8$ & 0,03 \\
\hline & Não & $30,8 \%(8)$ & $6,2 \%(2)$ & & & \\
\hline \multirow[t]{2}{*}{ Tempo de AME (meses) } & $\geq 4$ & $48 \%(12)$ & $61,3 \%(19)$ & 0,6 & $0,2-1,7$ & 0,32 \\
\hline & $<4$ & $52 \%(13)$ & $38,7 \%(12)$ & & & \\
\hline \multirow[t]{2}{*}{ AM 12 meses } & $\geq 12$ & $46,2 \%(12)$ & $40,6 \%(13)$ & 1,25 & $0,4-3,6$ & 0,67 \\
\hline & $<12$ & $53,8 \%(14)$ & $59,4 \%(19)$ & & & \\
\hline \multirow[t]{2}{*}{ AM 24 meses } & $=24$ & $11,5 \%(3)$ & $18,8 \%(6)$ & 0,6 & $0,1-2,5$ & 0,45 \\
\hline & $<24$ & $88,5 \%(23)$ & $81,2 \%(26)$ & & & \\
\hline
\end{tabular}

OR: razão de chances (odds ratio); IC: intervalo de confiança; M: masculino; F: feminino; C: cesárea; V: vaginal; AM: aleitamento materno; AME: AM exclusivo.

Tabela 2 - Análise de regressão linear múltipla tendo como variável dependente o grupo A

\begin{tabular}{llll}
\hline Variáveis independentes & OR ajustado & IC 95\% & p-valor \\
\hline 10 ou mais consultas médicas & 0,39 & $0,11-1,37$ & 0,133 \\
Tipo de parto cesárea & 3,47 & $1,03-11,66$ & 0,041 \\
AM antes de 6h de vida & 0,16 & $0,03-0,93$ & 0,025 \\
\hline
\end{tabular}

OR: razão de chances (odds ratio); IC: intervalo de confiança; AM: aleitamento materno.

0,01 e 0,03 respectivamente) (Tabela 2 ).

\section{DISCUSSÃO}

O grupo observado foi de crianças de 2 anos de idade, buscando-se estudar a influência de algumas variáveis referentes aos mil dias, da concepção até 24 meses de vida, no estado nutricional e desenvolvimento de excesso de peso em crianças nascidas a termo e assistidas por equipe multidisciplinar.

Ao comparar os grupos, observou-se que crianças nascidas de cesáreas tiveram 3,47 vezes mais chance de apresentar risco de sobrepeso e sobrepeso aos 2 anos de idade do que as nascidas de parto vaginal. O parto tipo cesárea predispõe a colonização da microbiota intestinal do neonato com micro-organismos patogênicos e configuração disbiótica, sendo um fator de risco 
para o desenvolvimento de doenças sistêmicas, como a obesidade. ${ }^{6,13}$

Na literatura, diversos estudos descreveram a diferença entre as composições das microbiotas fecais de neonatos nascidos de parto vaginal e cesárea, evidenciando a presença de uma microbiota obesogênica nos nascidos por cesárea. ${ }^{19,20} \mathrm{~A}$ associação entre parto do tipo cesárea e excesso de peso foi analisada no estudo transversal de Erigene Rutayisire e colaboradores com 8.900 crianças entre 3 e 6 anos, no qual a cesárea se mostrou significativamente associada ao risco de sobrepeso e de obesidade, mesmo após ajuste pelas variáveis de possível confundimento na regressão linear múltipla. ${ }^{21}$

Também se observou que crianças amamentadas antes de 6 horas de vida tiveram 6,25 vezes menos chance de apresentar risco de sobrepeso ou sobrepeso. O aleitamento materno antes de 6 horas de vida se relaciona com o estado nutricional de neonatos por meio da microbiota, assim como o tipo de parto. No entanto, o aleitamento materno precoce se mostra como um fator protetor ao excesso de peso, ao contrário do tipo de parto. Tendo como fatores principais o colostro e o contato pele-pele, essa prática promove a colonização simbiótica da mucosa intestinal e o estímulo à amamentação bem-sucedida e continuada. , $^{8,10}$

Mesmo em cenário de Hospital Amigo da Criança e esforços da equipe multidisciplinar durante os 2 anos de vida, não foi possível anular a influência de fatores referentes à cesárea e à ausência de aleitamento materno antes de 6 horas de vida. Tal achado revela que esses fatores tendem a ter um alto grau de influência a longo prazo no metabolismo da criança, com a possibilidade de predispor o excesso de peso até a adultícia.

As variáveis que revelaram ser estatisticamente significativas quando comparados grupos A e B têm grande influência da microbiota. Tanto o tipo de parto quanto o aleitamento materno precoce nas primeiras horas de vida têm como possível elo com o risco de sobrepeso e sobrepeso a modulação da microbiota. Para comprovar esta hipótese, seria interessante a análise laboratorial da microbiota dos neonatos em momentos oportunos para evidenciar alterações geradas por essas variáveis referentes ao nascimento e ao período pós-natal sobre a composição dos micro-organismos.

O não aleitamento materno se relacionou positivamente com o uso de fórmula na maternidade. Isso quer dizer que recém-nascidos que não foram aleitados antes de 6 horas de vida têm uma grande possibilidade de ter recebido fórmula láctea na maternidade. Não foi encontrada associação entre o tipo de parto e o aleitamento precoce, contrariando trabalhos na literatura que revelam que a cesárea se mostra como uma barreira para a iniciação da amamentação e do aleitamento nas primeiras horas de vida do neonato. ${ }^{22-24}$ Essa barreira pode ser explicada pelo fato de que o parto do tipo cesárea é um procedimento cirúrgico, tendo um tempo de recuperação maior em comparação com o parto vaginal. ${ }^{25}$ Consequentemente, a tendência do neonato não ser amamentado precocemente é maior, sendo uma barreira para a iniciação da amamentação e um fator prejudicial para a instalação de relação íntima mãe-neonato, que poderia contribuir para um padrão de amamentação duradouro..$^{10-26}$

\section{CONCLUSÕES}

Os resultados do presente trabalho mostram o impacto do tipo de parto e do aleitamento materno precoce em um aspecto da saúde da criança que é de grande relevância.

A análise das variáveis demonstra que o estado antropométrico das crianças aos 24 meses de vida é resultado da modulação de fatores relacionados ao período pré-natal, ao parto e à alimentação infantil. Sendo assim, o período que se estende da concepção até os 24 meses da criança deve ser valorizado e estudado para que suas possíveis modulações sejam melhor exploradas visando à prevenção de agravos e de doenças crônicas.

\section{REFERÊNCIAS}

1 Ministério da Saúde. Sistema de Vigilância Alimentar e Nutricional. Relatório do Estado Nutricional dos indivíduos acompanhados por período, fase do ciclo da vida e índice. 2017 [acesso em $17 \mathrm{dez} 2018$ ]. Disponível em: http://dabsistemas. saude.gov.br/sistemas/sisvanV2/relatoriopublico/index

2 Bhutta ZA, Ahmed T, Black RE, Cousens S, Dewey K, Giugliani $\mathrm{E}$ et al. What works? Interventions for maternal and child undernutrition and survival. Lancet. 2008;371(9610):417-40. DOI: https://doi.org/10.1016/S0140-6736(07)61693-6

3 Wopereis H, Oozeer R, Knipping K, Belzer C, Knol J.

The first thousand days-intestinal microbiology of early life: establishing a symbiosis. Pediatr Allergy Immunol. 2014;25(5):428-38.

4 Alexander DD, Yan J, Bylsma LC, Northington RS, Grathwohl $D$, Steenhout P et al. Growth of infants consuming wheypredominant term infant formulas with a protein content of $1.8 \mathrm{~g} / 100 \mathrm{kcal}:$ a multicenter pooled analysis of individual participant data. Am J Clin Nutr. 2016;104(4):1083-92.

5 Wang M, Monaco MH, Donovan SM. Impact of early gut microbiota on immune and metabolic development and 
function. Semin Fetal Neonatal Med. 2016;21(6):380-7.

6 Walker A. Breast milk as the gold standard for protective nutrients. J Pediatr. 2010;156:(2 Suppl):S3-7.

7 Palmeira P, Carneiro-Sampaio M. Immunology of breast milk. Rev Assoc Med Bras. 2016;62(6):584-93. http://dx.doi. org/10.1590/1806-9282.62.06.584

8 World Health Organization. Early initiation of breastfeeding to promote exclusive breastfeeding. Genebra (SZ) [revised on Fev 20, 2018; cited on Mar 30, 2018]. Available from: http://www. who.int/elena/titles/early_breastfeeding/en/

9 Moore ER, Bergman N, Anderson GC, Medley N. Early skinto-skin contact for mothers and their healthy newborn infants. Cochrane Database Syst Rev. 2016;11. DOI: https://doi. org/10.1002/14651858.CD003519.pub4

10 Ministério da Saúde. Secretaria de Atenção à Saúde. Departamento de Atenção Básica. Saúde da criança: nutrição infantil - aleitamento materno e alimentação complementar. Caderno de Atenção Básica, n. 23. Brasília: MS; 2009. 112p.

11 Sutharsan R, Mannan M, Doi SA, Mamun AA. Caesarean delivery and the risk of offspring overweight and obesity over the life course: a systematic review and bias-adjusted metaanalysis. Clin Obes. 2015;5(6):293-301. DOI: https://doi. org/10.1111/cob.12114

12 Dominguez-Bello MG, Costello EK, Contreras M, Magris M, Hidalgo G, Fierer N et al. Delivery mode shapes the acquisition and structure of the initial microbiota across multiple body habitats in newborns. Proc Natl Acad Sci USA. 2010;107(26):11971-5.

13 Cabrera-Rubio R, Collado MC, Laitinen K, Salminen S, Isolauri E, Mira A. The human milk microbiome changes over lactation and is shaped by maternal weight and mode of delivery. Am J Clin Nutr. 2012;96(3):544-51. DOI: https://doi.org/10.3945/ ajcn.112.037382

14 Lamounier JA. Promoção e incentivo ao aleitamento materno: iniciativa Hospital Amigo da Criança [acesso em 9 dez 2018]. J Pediatr (Rio J). 1996;72(6)363-8. Disponível em: http://www. jped.com.br/conteudo/96-72-06-363/port.pdf

15 Ministério da Saúde. Orientações para coleta e análise de dados antropométricos em serviços de saúde: norma técnica do Sistema de Vigilância Alimentar e Nutricional [acesso em 9 dez 2018]. Brasília: MS; 2011. Disponível em: http://dab. saude.gov.br/portaldab/biblioteca.php?conteudo=publicacoes $/$ orientacoes_coleta_analise_dados_antropometricos

16 Chaves RG, Lamounier JA, César CC. Factors associated with duration of breastfeeding. J Pediatr (Rio J). 2007;83(3):241-6. http://dx.doi.org/10.1590/S0021-75572007000400009

17 Ministério da Saúde. Saúde da criança: crescimento e desenvolvimento [acesso em 9 dez 2018]. Caderno de Atenção Básica, n. 33. Brasília: MS; 2012. Disponível em: http://bvsms. saude.gov.br/bvs/publicacoes/saude_crianca_crescimento_ desenvolvimento.pdf

18 Sociedade Brasileira de Pediatria. Departamento de Pediatria Ambulatorial. Manual Prático de Atendimento em Consultório e Ambulatório de Pediatria [acesso em 9 dez 2018]. SBP; 2006. Disponível em: http://www.sbp.com.br/fileadmin/user upload/2015/02/ManPraticaAtend.pdf

19 Penders J, Thijs C, Vink C, Stelma FF, Snijders B, Kummeling I et al. Factors influencing the composition of the intestinal microbiota in early infancy [accessed on Dez 9, 2018]. Pediatrics. 2006;118(2):511-21. Available from: http://pediatrics.aappublications.org/content/118/2/511. long?sso $=1 \delta$ sso_redirect_count $=1 \delta$ nfstatus $=401$ \&nftok en $=00000000-0000-0000-0000-000000000000 \delta$ nfstatusdes cription $=$ ERROR $\% 3 a+N o+$ local + token

20 Huurre A, Kalliomaki M, Rautava S, Rinne M, Salminen S, Isolauri E. Mode of delivery: effects on gut microbiota and humoral immunity [accessed on Dez 9, 2018]. Neonatology. 2008;93(4):236-40. Available from: https://www.karger.com/?DOI=10.1159/000111102

21 Rutayisire E, Wu X, Huang K, Tao S, Chen Y, Tao F. Cesarean section may increase the risk of both overweight and obesity in preschool children. BMC Pregnancy Childbirth. 2016;16:338. DOI: https://doi.org/10.1186/s12884-016-1131-5

22 Batal M, Boulghaurjian C. Breastfeeding initiation and duration in Lebanon: are the hospitals "mother friendly" [accessed on Dez 9, 2018]. J Pediatr Nurs. 2005;20(1):53-9. Available from: https://Www.pediatricnursing.org/article/ S0882-5963(04)00136-8/fulltext

23 Pérez-Escamilla R, Maulen-Radovan I, Dewey K. The association between cesarean delivery and breast-feeding outcomes among Mexican women [accessed on Dez 9 2018]. Am J Public Health. 1996;86(6):832-6. Available from: https://www.ncbi.nlm.nih.gov/pmc/articles/PMC1380403/ pdf/amjph00517-0070.pdf

24 Theofilogiannakou M, Skouroliakou M, Gounaris A, Panagiotakos D, Markantonis S. Breast-feeding in Athens, Greece: factors associated with its initiation and duration [accessed on Dez 9, 2018]. J Pediatr Gastroenterol Nutr. 2006;43(3):379-84. Available from: https://insights.ovid. com/pubmed?pmid $=16954963$

25 Pérez-Ríos N, Ramos-Valencia G, Ortiz AP. Cesarean delivery as a barrier for breastfeeding initiation: the Puerto Rican experience. J Hum Lact. 2008;24(3):293-302. DOI: https://doi. org/10.1177/0890334408316078

26 Hobbs AJ, Mannion CA, McDonald SW, Brockway M, Tough SC. The impact of caesarean section on breastfeeding initiation, duration and difficulties in the first four months postpartum. BMC Pregnancy Childbirth. 2016;16:90. DOI: https://doi. org/10.1186/s12884-016-0876-1 